\title{
IMPACTOS DA PANDEMIA DA COVID-19 EM PESSOAS COM DOENÇA DE PARKINSON ACOMPANHADAS POR TELEMONITORAMENTO
}

Carlos Renê Silva Miranda Filho; Universidade Federal de Pernambuco; carlos.rene@ufpe.br;

Luis Felipe Pohlmann Tabarelli; Universidade Federal de Pernambuco; luis.tabarelli@ufpe.br; João Marcelo Duarte Ribeiro Sobrinho*; Universidade Federal de Pernambuco; joao.mdrsobrinho@ufpe.br;

David Duarte Timponi França; ; Universidade Federal de Pernambuco; david.timponi@ufpe.br; Amdore Guescel C Asano; Hospital das Clínicas; Universidade Federal de Pernambuco; amdore.asano@ufpe.br; Maria das Graças Wanderley de Sales Coriolano; Programa de Pós-Graduação em Gerontologia; Universidade Federal de Pernambuco; mariagracas.coriolano@ufpe.br;

Nadja Maria Jorge Asano; Programa de Pós-Graduação em Gerontologia; Universidade Federal de Pernambuco; nadja.asano@ufpe.br.

\section{RESUMO}

Introdução: Durante a pandemia da COVID-19 foram adotadas medidas de isolamento social, para reduzir o contágio pelo novo coronavírus. Essas determinações dificultaram o acesso a cuidados médicos, exercícios e terapias, necessários para pessoas com Doença de Parkinson (DP). Objetivos: Esse trabalho busca entender como a pandemia e o isolamento social podem influenciar a sintomatologia da DP e as dificuldades enfrentadas pelos pacientes. Métodos: Pessoas com DP cadastradas no Programa Pró-Parkinson da Universidade Federal de Pernambuco foram convidadas por telefone para o telemonitoramento. Foram realizadas entrevistas, de 01/07/2020 a 25/08/2020. Na avaliação das barreiras na realização de atividades de vida diária foi aplicado MDS-UPDRS parte II: Aspectos Motores de Experiências da Vida Diária (M-EVD). Resultados: Foram acompanhadas 31 pacientes, com idade média de 67 anos. 64,5\% dos indivíduos relataram piora dos sintomas, entre esses $40 \%$ apresentaram dificuldades de adesão ao tratamento farmacológico. Foi constatado que 90,3\% apresentaram, como uma barreira importante, o acesso à fisioterapia durante a pandemia. Piora da marcha $(67,7 \%)$, ou fala $(61,3 \%)$ e dificuldade na execução de tarefas cotidianas foram as atividades de maior desconforto durante isolamento. Conclusão: Através do telemonitoramento observou-se que a pandemia da Covid-19 afetou significativamente a sintomatologia e o manejo clínico das pessoas com DP, ao distanciá-las dos serviços médicos e terapêuticos. Além disso, estresse e ansiedade durante o isolamento possivelmente contribuíram para a piora dos sintomas motores e não motores da doença. $\mathrm{O}$ telemonitoramento teve importante contribuição para o acompanhamento durante o isolamento.

Palavras-chave: Doença de Parkinson; Infecções por coronavirus; Telemonitoramento; Isolamento social.

Agradecimentos: *À Pró-Reitoria de Extensão e Cultura da Universidade Federal de Pernambuco, edital 2020-06 PIBEXC, pelo financiamento do projeto de extensão, PRÓ-PARKINSON - AÇÕES MULTIDISCIPLINARES NO ENFRENTAMENTO DA COVID-19. 